\title{
Laboratory Measurement Campaign of DVB-T Signal With Transmit Delay Diversity
}

\author{
Raffaele Di Bari, Maurice Bard, Yue Zhang, Karim Medhat Nasr, John Cosmas, Kok-Keong Loo, \\ Rajagopal Nilavalan, Hamidreza Shirazi, and Kannan Krishnapillai
}

\begin{abstract}
The requirements for future DVB-T/H networks demand that broadcasters design and deploy networks that provide ubiquitous reception in challenging indoors and other obstructed situations. It is essential that such networks are designed cost-effectively and with minimized environmental impact. The EC funded project PLUTO has since its start in 2006 explored the use of diversity to improve coverage in these difficult situations. The purpose of this paper is to investigate the performance of Transmit Delay Diversity (DD) with two antennas to improve the reception of DVB-T/H systems operating in different realistic propagation conditions through a series of tests using a SPIRENT SR5500 dual channel emulator. The relationship between correlation coefficient between channels, receiver velocity and diversity gain is investigated. It is shown that transmit delay diversity significantly improves the quality of reception particularly in simulated fast fading mobile broadcasting applications. This paper documents research conducted by Brunel University and Broadreach Systems.
\end{abstract}

Index Terms-Doppler frequency, DVB-T/H, errored second ratio (ESR), MIMO, spatial correlation, transmit delay diversity (DD).

\section{INTRODUCTION}

W HEREAS current broadcast TV networks have been designed to enable reception by rooftop antennas, next generation digital TV service providers for mobile and fixed reception are finding that coverage indoors and areas of non Line Of Sight (LOS) is a fundamental requirement. It is essential that

Manuscript received September 23, 2007; revised March 31, 2008. First published July 9, 2008; last published August 20, 2008 (projected).

R. Di Bari, K.-K. Loo, R. Nilavalan, and K. Krishnapillai are with the Department of Electronic and Computer Engineering, School of Engineering and Design, Brunel University, Uxbridge, London, UB8 3PH, U.K. (e-mail: raffaele.dibari@brunel.ac.uk; jonathan.loo@brunel.ac.uk; Rajagopal.Nilavalan@brunel.ac.uk; kannan.krishnapillai@brunel.ac.uk).

M. Bard is with Broadreach Communications Ltd, Torquay TQ1 1QB,U.K. (e-mail: mail@broadreachSystems.com).

Y. Zhang is with the Department of Electronic and Computer Engineering, School of Engineering and Design, Brunel University, Uxbridge, London, UB8 3PH, U.K.. He is also with Anritsu Company, Rutherford Close Stevenage, Hertfordshire SG1 2EF,U.K. (e-mail: Yue_Zhang @ ieee.org).

K. M. Nasr is with BBC Research British Broadcasting Corporation, Tadworth, Surrey KT20 6NP, U.K. (e-mail: karim.nasr@rd.bbc.co.uk).

J. Cosmas is with the Networks and Multimedia Communications Group, The Department of Electronic and Computer Engineering, School of Engineering and Design, Brunel University, Uxbridge, London UB8 3PH, U.K. (e-mail: John.cosmas@brunel.ac.uk).

H. Shirazi is with Multimedia and Networking Communications Group, School of Engineering and Design, Brunel University, Uxbridge, Middlesex UB8 3PH, U.K. (e-mail: Hamidreza.Shirazi@brunel.ac.uk; http://people.brunel.ac.uk/ eepghhs).

Color versions of one or more of the figures in this paper are available online at http://ieeexplore.ieee.org.

Digital Object Identifier 10.1109/TBC.2008.2000462 such networks are designed cost-effectively and with minimized environmental impact. The EC funded project PLUTO [1] has since its start in 2006 explored the use of diversity and low delay on-channel repeaters to improve coverage in these difficult situations.

The use of transmit diversity techniques with multiple antennas have long been proposed to improve the performance and capacity of wireless systems [2], [3]. Transmit diversity exploits the scattering effect inherent in the channel by means of transmitting multiple signals in a controlled manner from spatially separated antennas. This allows independently faded signals to arrive at the receiver and improves the chances of decoding a signal of acceptable quality. Although transmit diversity is a commonly used technique for most wireless communication systems such as mobile telephony and WLAN, (e.g. GSM, UMTS, IEEE 802.11/a,b,g,n), it has not been applied for DVB-T/H or DAB systems. The main advantage of Transmit Diversity over other MIMO techniques is that it can be applied to standards based systems such as DVB-T/H, DAB/DMB without modification. Transmit diversity can complement receive diversity by adding an additional diversity gain and in situations where receiver diversity is not practical, transmit diversity alone delivers a comparable amount of diversity gain.

In Delay Diversity (DD), the same information is transmitted from both antennas simultaneously but with a delay of several OFDM symbol intervals $\mathrm{T}$, to overcome the effects of flat fading. Spatial transmit diversity can be implemented by transmitting identical signals from different transmit antennas, which are spatially separated from each other. This spatial separation of transmit antennas is required to achieve a sufficient de-correlation of the channels. Modeling predicts that a minimum separation of approximately 10 times the carrier frequency wavelength is required for a typical urban transmitter. In LOS or near LOS conditions, the respective signal paths between spatially separated antennas and the mobile receiver are likely to be correlated to a certain degree because of insufficient multipath in the channel. This results in a diversity loss and methods of overcoming this are also being researched.

This paper documents measurement made using actual receiver equipment in simulated laboratory conditions. The laboratory set-up includes a DVB modulator, a wireless channel emulator and a Broadreach Systems Monitor Station. The Monitor Station is an equipment developed by Broadreach Systems that comprises a standard DVB receiver and additional processing to enable engineering data to be extracted. The Monitor Station act in exactly the same was as a standard set-top box receiver. The objective of the lab tests is to confirm that diversity gains 


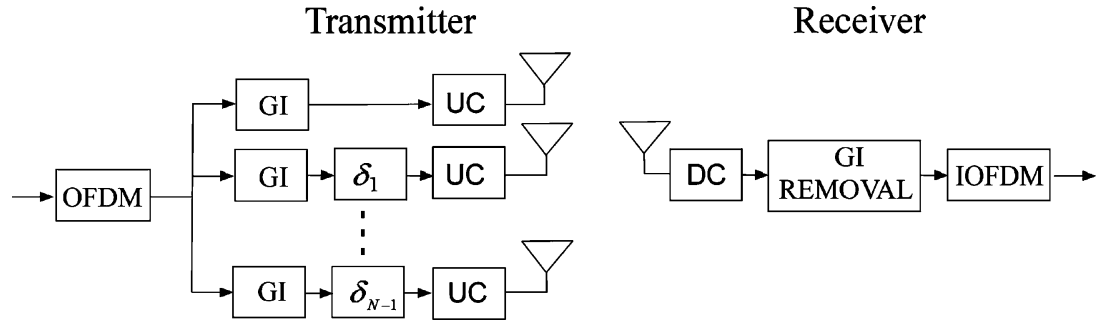

Fig. 1. Transmit delay diversity applied to an OFDM system.

reported in the literature [7], [10], [11] predicted by mathematical modeling can be realized in realistic conditions using actual equipment.

A series of experiments were conducted to measure the dependence of transmit diversity gain against:

- Modulation scheme

- Diversity Delay

- Cross correlation coefficient

- Doppler shift or speed of the receiver

- Channel profile; indoors, outdoors etc.

This paper is organized as follows. Section II introduces a theoretical formulation of delay diversity applied to the DVB signals. Section III describes the laboratory set-up for diversity testing and evaluation. Section IV describes the measurement methodology, the channel models used for testing as well as the main system parameters and a summary of test cases. Section V presents the measurement results and analyses of the different studied cases. Finally, Section VI concludes the paper.

\section{TRAnSmit Delay Diversity}

DD consists of transmitting a main signal and its delayed replica through two or more antennas. Fig. 1 shows the block diagram of $\mathrm{N}$-transmit antennas applied to an OFDM system with DD. The OFDM modulated signals are transmitted using $\mathrm{N}$ antennas. The individual signals only differ in an antenna specific delay shift $\delta$. After the insertion of the cyclic prefix or Guard Interval (GI), the delay $\delta$ is inserted. The functional blocks "UC" and "DC" stand for up-conversion and down-conversion, respectively, of the signals from the baseband into RF-band and vice versa.

DD can be applied to a standard DVB-T/H implementation because its signal processing is performed on the OFDM output symbols in the time-domain. Consider that the transmitted symbols are denoted by $s(t), t=0, \ldots K-1$ where $K$ is the number of subcarriers. During transmission, these symbols are delayed between respective $\mathrm{N}$ transmit antennas. The symbols transmitted from $i^{t h}$ antenna at time $t$ can be represented by:

$$
s_{i}(t)=s_{i}\left(t-\delta_{i}\right) v\left(t-\delta_{i}\right)
$$

where $v(x)$ is a mathematical function that sets zeros for negative time indices (see Appendix), $i=0,1, \ldots, N-1$, and $\delta_{i}$ is the delay introduced between the symbols. The delayed symbols can be recorded in a diagonal matrix as a codeword, $\mathrm{C}$ defined below:

$$
C=\left(\begin{array}{cccc}
s(0) & s(1) & \ldots & s(K-1) \\
0 & s(0) & \ldots & s(K-2) \\
\ldots & \ldots & \ldots & \ldots \\
0 & 0 & \ldots & s(K-N)
\end{array}\right)_{N \times K}
$$

From (1) and (3), it can be seen that $i^{\text {th }}$ antenna is actually transmitting $s(0), s(1), \ldots, s(K-1)$ sequence that is delayed by $\delta_{i}$. This can be then denoted as $0, \ldots, s(0), s(1), \ldots s\left(K-1-\delta_{i}\right)$. This system is equivalent to the transmission of the sequence $s(0), s(1), \ldots s(K-1)$ over a propagation channel (given by (3))with one transmit antenna to receiver

$$
h^{e, t}(d)=\sum_{i=1}^{N} h_{i}^{t}\left(d-\delta_{i}\right) v\left(d-\delta_{i}\right)
$$

where $h^{e, t}$ is the equivalent channel impulse response (CIR) at the receiver side, $t$ denotes that $\mathrm{CIR}$ is in time domain, $\mathrm{d}$ denotes the index of delay in channel delay profile, and $\mathrm{N}$ denotes the number of transmitter antennas.

Therefore the CIR can be described as

$$
h^{e, t}(d)=\left\lfloor h^{e, t}(0), \ldots, h^{e, t}(K-1)\right\rfloor
$$

In the frequency domain, the equivalent channel transfer function is expressed as:

$$
H^{e}(k)=\sum_{i=1}^{N} e^{-j \frac{2 \pi}{K} k \delta_{i}} H_{i}(k)
$$

where according to [10], $H_{i}(k)$ denotes the channel frequency response of the transmission from the $i^{t h}$ transmit antenna to the receive antenna and $\delta_{i}$ is the delay of the $i^{t h}$ transmit antenna (there is no delay at first transmit antenna, $\delta_{1}=0$ ).

Fig. 2 shows the channel frequency response measured from a spectrum analyzer for a DVB-T system with and without DD. It is clear that DD has transformed the spatial diversity into frequency diversity; in other words the Multiple Input Single Output (MISO) channel is transformed into a single-input single-output (SISO) channel with increased frequency-selectivity. Therefore, the coherence bandwidth is decreased. In Section VI, experimental results show that a lower coherence 

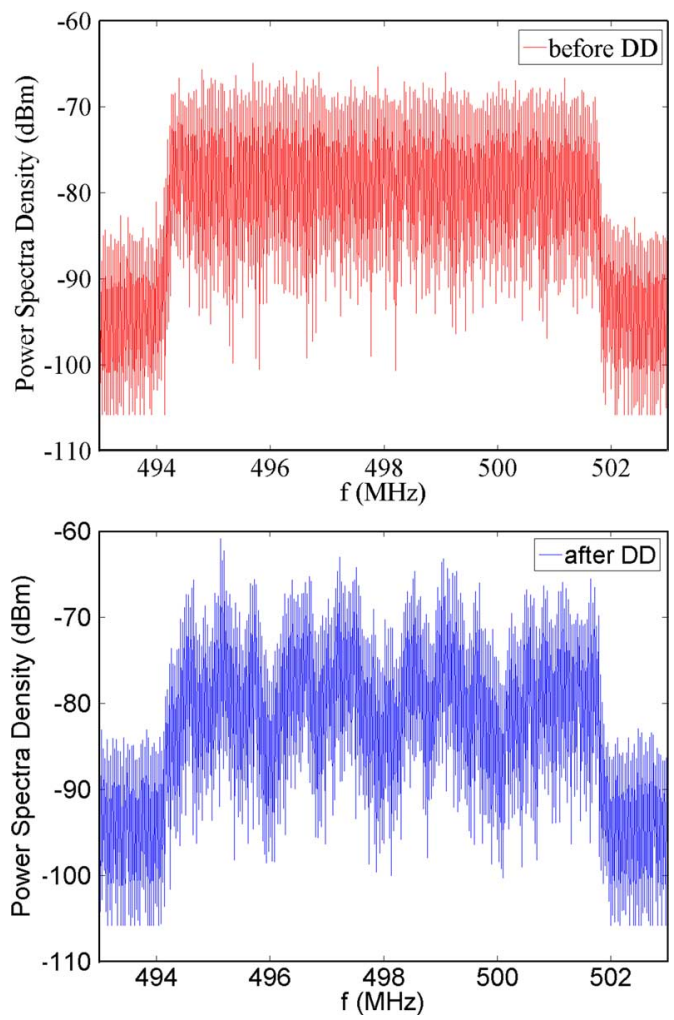

Fig. 2. Power spectra density of signal before and after DD UHF (498 MHz).

bandwidth leads to a better error performance for coded OFDM systems in multipath environments.

As shown in Fig. 1, the cyclic prefix should be added before the delay operations. No Intersymbol Interference (ISI) occurs if:

$$
\delta_{i} \leq N_{g}-\tau_{\max }, \quad i=0,1, \ldots N-1
$$

where $N_{g}$ and $\tau_{\max }$ are the guard interval length and maximum channel delay respectively.

If this condition stands, it can also be proven that $\mathrm{DD}$ is equivalent to other diversity techniques, e.g. Cyclic Delay Diversity (CDD) and Phase Delay (PD) [7], [10]. To achieve any diversity effects (i.e. to obtain constructive and destructive interference within the OFDM signal bandwidth B), the delays $\delta_{i}$ (in samples) have to fulfill the relation

$$
\delta_{i} \geq \frac{1}{B \cdot T_{S}}
$$

where Ts denotes the sampling time of the OFDM time domain signal. Results reported in [7] show that a delay of $\delta_{i} \geq 1.5 \mu \mathrm{s}$ derives no further improvement of diversity gain, defined as the offset between the $\mathrm{C} / \mathrm{N}$ needed to achieve the same performances in single and multiple diversity antennas system.

In [11], it was shown that diversity gain is fully effective over uncorrelated channels. However, in reality, the respective signal paths between spatially separated antennas and the mobile receiver are likely to be correlated to a certain degree because of insufficient antenna separation at the transmitter and the lack of sufficient multipath in the channel. Table I
TABLE I

RELATIONSHIP BETWEEN CHANNEL CORRELATION AND TRANSMITTER ANTENNAS SEPARATION

\begin{tabular}{lc}
\hline \hline $\begin{array}{l}\text { Correlation between two } \\
\text { transmitter antenna }(\rho)\end{array}$ & $\begin{array}{l}\text { Distance between two } \\
\text { transmitter antenna }(\lambda \text { wavelength })\end{array}$ \\
\hline 1 & 0 \\
0.75 & 6.5 \\
0.50 & 9.7 \\
0.25 & 13 \\
0 & 17.5 \\
\hline
\end{tabular}

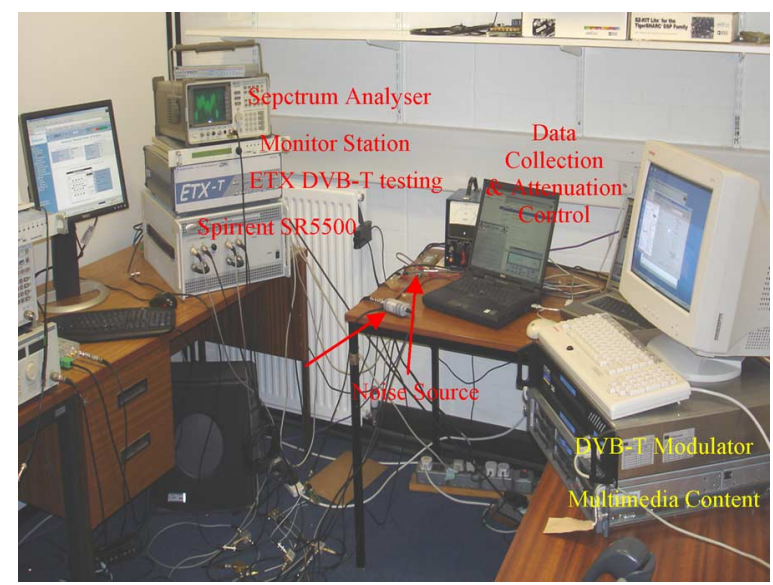

Fig. 3. Laboratory test bench facilities.

shows the relationship between the channel correlation coefficient, $\rho$, and the transmitter antennas separation expressed in wavelength $\lambda$ [12]. The degree of channel correlation as in $\rho=\{0,0.25,0.5,0.75,1\}$ corresponds to separation distances between two transmit antennas. We assume that spatial fading correlation occurs at the transmitter and thus the correlated signals appear at the receiver. In this case, the correlated channel is expressed as $h_{2}=\rho \cdot h_{1}+\sqrt{1-\rho^{2}} w^{2}$ where $h_{1}$ and $h_{2}$ are the two correlated fading channels, $w$ is the Rayleigh fading factor and $\rho$ is the correlation coefficient between $h_{1}$ and $h_{2}$. For $\rho=0$, the two fading channels are fully uncorrelated while $\rho=1$ represents a fully correlated case. The cross correlation between the two channels results in diversity loss and performance degradation of DD. Thus, it is apparent that DD cannot achieve the same diversity gain as obtained under the uncorrelated channel [11]. Therefore, the relationships linking the diversity gain, delays and the cross correlation of the channels are of high importance and are thus studied in this paper through the laboratory setup described in Section III.

\section{LABORATORY SET-UP FOR TRANSMIT DELAy DiVERSITY TESTING}

This section describes the laboratory simulation set-up as depicted in Figs. 3 and 4. A test signal was generated by a standard DVB-T modulator, the output signal was then split into two paths and fed directly into a Spirent SR5500 radio channel emulator. Testing was conducted in the UHF band at channel 24 (498 MHz).

The Spirent SR5500 [13] is a two-path wireless channel emulator that simulates the characteristics of complex wideband radio channels, including time-varying multipath delay spread, 


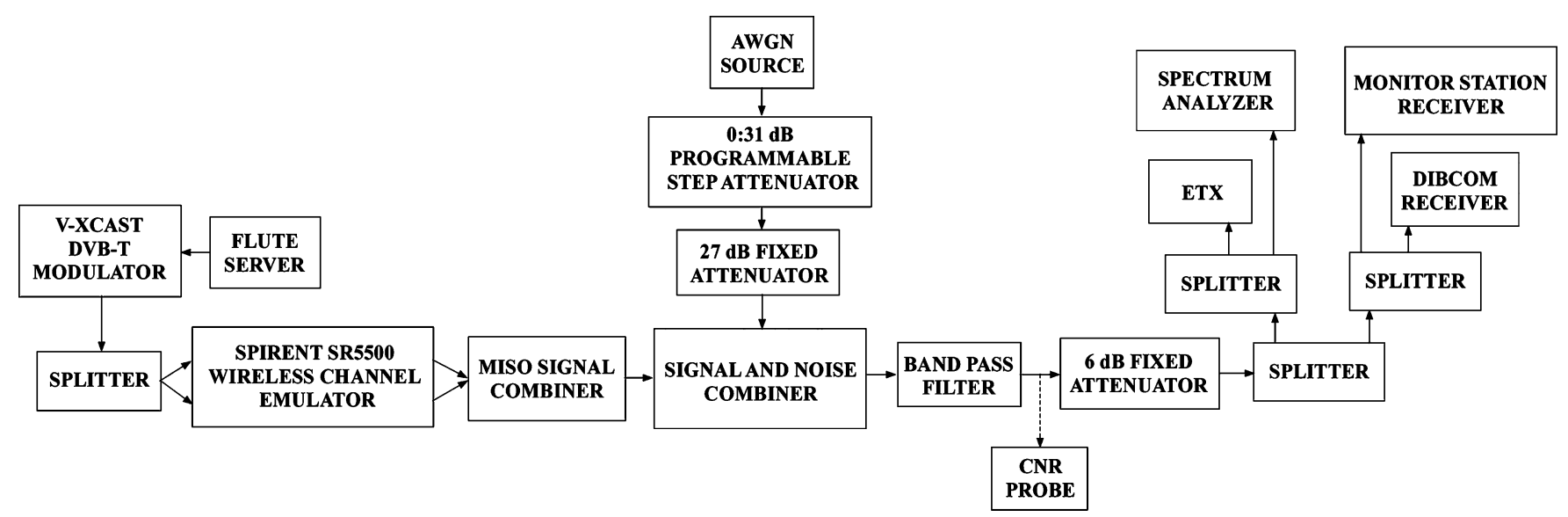

Fig. 4. Block diagram's of laboratory test bench.

fast and slow fading, path loss and variable amounts of correlation between the paths. The SR5500 allows the generation of arbitrary power delay profiles to emulate different channel scenarios (e.g. outdoor, indoor, and vehicular). Varying the correlation coefficient and the delay between the channels allows the investigation of the effect of diversity in realistic and practical channel conditions.

The output signals were combined to simulate the signal at a single receive antenna. Additive White Gaussian Noise (AWGN) was generated by a variable noise source and added to the main signal to vary Carrier to Noise ratio at the receiver input. The nominal noise flatness of the noise generator was $+/-2 \mathrm{~dB}$ over $1 \mathrm{GHz}$ bandwidth. A band-pass filter was used to cut off the out-of-band noise, preventing saturation of the receiver broadband amplifier. Additional fixed attenuators were used to adjust the power to be within the dynamic range of a number of receiver devices such as the Rhode and Schwartz ETX DVB-T testing unit, the Dibcom DVB-T/H receiver and the Broadreach monitoring station. A splitter finally fed the faded signals into a spectrum analyzer and the three receivers. The results published here are for the Broadreach monitor Station which being based on a standard DVB set top box receiver provided performance most representative of general purpose DVB receivers.

\section{Measurement Methodology, Channel Models and MAIN SYSTEM PARAMETERS}

\section{A. Measurement Methodology}

Testing was performed with 2 simulated transmit antennas. The delay value $\delta$ between the two signals was introduced by the Spirent channel emulator. In real word applications, the delay between signals would be introduced by a dedicated device or by using a specialized modulator designed specifically for transmit diversity. It is important to note that the overall transmitted power was normalized for all measurements, i.e. either $100 \%$ of the power was transmitted through a single antenna or $50 \%$ of the power was transmitted by each of 2 antennas. It was found to be impractical to base our measurements on the normally used BER metrics due to the bursty nature of the errors, and hence a criterion called ESR (Errored Seconds Ratio) was defined. An errored second occurs when the receiver counts at least one MPEG Transport Stream errored frame within a given second, e.g. an uncorrectable Reed-Solomon codeword. The ESR is the percentage of errored seconds observed during a defined integration period, this period being selected to be long enough to average effects of short term fading. Generally a duration of 20 minutes was found to be adequate for most scenarios and rendered a stable and accurate measure. The ESR was measured as a function of $\mathrm{C} / \mathrm{N}$ at the receiver input so that we were able to measure the $\mathrm{C} / \mathrm{N}$ at an arbitrary Threshold of Visibility (ToV) of $3 \%$. This is equivalent to 2 errored seconds every minute [14].

To determine the ESR, the signal power was kept at a constant level at least $20 \mathrm{~dB}$ above the receiver's noise floor, and the level of additive excess noise increased by changing the step attenuator, as recommended in [15]. The signal power and the noise level were measured after the Signal and Noise combiner using a power meter. A simple calculation is used to map each ESR point to its corresponding $\mathrm{C} / \mathrm{N}$. The power meter had been set-up with 4096 points to establish the average, corresponding to 5 minutes of power integration in the time domain. The measurements precision was estimated to be within $0.5 \mathrm{~dB}$.

\section{B. Channel Models}

Tests were performed using 4 different simulated scenarios. These were the Indoor Modified "Commercial B", [16] and COST 207 [18] outdoor environments: Rural Area (RA), Typical Urban (TU) and Bad Urban (BU) in UHF band. The Indoor Modified "Commercial B" is a derivation of the channel delay profile, which is specified by ITU-R [16]. The standard scenario was been "modified" with the addition of 2 short delay taps (at 15 and $25 \mathrm{~ns}$ ) to make the model more realistically represent reflections from nearby objects in an indoor scenario. The 2 additional taps have the effect of introducing significant 'flat fading'.

Table II gives the values of the tap delays, the associated mean powers of all profiles, the Root Mean Square (RMS) delay spread $\sigma_{\tau}$ and the associated approximate $90 \%$ coherence bandwidth, calculated according [17]. All the channels have Rayleigh fading. The power delay profiles are generated within the Spirent channel emulator and in the case of two transmit 
TABLE II

ChanNel MODELS USED FOR TESTING

\begin{tabular}{|c|c|c|c|c|c|c|c|c|}
\hline \multirow[b]{2}{*}{ Taps } & \multicolumn{2}{|c|}{ Indoor } & \multicolumn{2}{|c|}{$\mathrm{RA}$} & \multicolumn{2}{|c|}{ TU6 } & \multicolumn{2}{|c|}{ BU12 } \\
\hline & $\begin{array}{c}\text { Relative } \\
\text { delay } \\
\text { (ns) }\end{array}$ & $\begin{array}{l}\text { Fading } \\
(\mathrm{dB})\end{array}$ & $\begin{array}{c}\text { Relative } \\
\text { delay } \\
\text { (ns) }\end{array}$ & $\begin{array}{l}\text { Fading } \\
(\mathrm{dB})\end{array}$ & $\begin{array}{c}\text { Relative delay } \\
\text { (ns) }\end{array}$ & $\begin{array}{l}\text { Fading } \\
\text { (dB) }\end{array}$ & $\begin{array}{c}\text { Relative } \\
\text { delay } \\
\text { (ns) }\end{array}$ & $\begin{array}{l}\text { Fading } \\
(\mathrm{dB})\end{array}$ \\
\hline 1 & 0 & -4.6 & 0 & -4.6 & 0 & -3.0 & 0 & -7 \\
\hline 2 & 15 & -2.0 & 100 & 0 & 100 & 0 & 200 & -3 \\
\hline 3 & 25 & -3.0 & 200 & -4.3 & 200 & -2.0 & 400 & -1 \\
\hline 4 & 50 & 0 & 300 & -6.5 & 300 & -6.0 & 800 & 0 \\
\hline 5 & 150 & -4.3 & 500 & -3.0 & 500 & -8.0 & 1600 & -2 \\
\hline 6 & 225 & -6.5 & 700 & -15.2 & 700 & -10.0 & 2200 & -6 \\
\hline 7 & 400 & -3.0 & - & - & - & - & 3200 & -7 \\
\hline 8 & 525 & -15.2 & - & - & - & - & 5000 & -1 \\
\hline 9 & 750 & -21.7 & - & - & - & - & 6000 & -2 \\
\hline 10 & - & - & - & - & - & - & 7200 & -7 \\
\hline 11 & - & - & - & - & - & - & 8200 & -10 \\
\hline 12 & - & - & - & - & - & - & 10000 & -15 \\
\hline $\begin{array}{l}\text { RMS Delay spread } \\
(\mu \mathrm{s})\end{array}$ & \multicolumn{2}{|c|}{0.1} & \multicolumn{2}{|c|}{0.1} & \multicolumn{2}{|c|}{1.1} & \multicolumn{2}{|c|}{2.5} \\
\hline $\begin{array}{c}90 \% \text { Coherence } \\
\text { bandwidth }(\mathrm{MHz})\end{array}$ & \multicolumn{2}{|c|}{0.2} & \multicolumn{2}{|c|}{0.2} & \multicolumn{2}{|c|}{0.0182} & \multicolumn{2}{|c|}{0.003} \\
\hline
\end{tabular}

TABLE III

DVB-T SYSTEM PARAMETERS

\begin{tabular}{|c|c|c|c|c|}
\hline Configuration Name & UK & & 4-QAM UK & French \\
\hline Code Rate & $3 / 4$ & & $3 / 4$ & $1 / 2$ \\
\hline Signal Constellation & 16-QAM & & 4-QAM & 64-QAM \\
\hline DVB-T Mode & & $2 \mathrm{k}$ & & $8 \mathrm{k}$ \\
\hline Hierarchy $(\alpha)$ & & \multicolumn{3}{|c|}{ Non hierarchical } \\
\hline Guard Interval & & \multicolumn{3}{|c|}{$1 / 32$} \\
\hline
\end{tabular}

antenna testing, the amount of correlation between channels can be varied.

\section{DVB System Parameters}

Basic transmission parameters are defined for an $8 \mathrm{MHz}$ channel by the DVB-T standard [19]. In the $2 \mathrm{k}$ mode (used in the UK), the OFDM symbol duration is $T_{U}=224 \mu \mathrm{s}$ and the number of carriers is $K=1705$. This yields a subcarriers spacing of $1 / T_{U}=4464 \mathrm{~Hz}$ and a spacing between the spectrum band edge carriers of $(K-1) / T_{U}=7.61 \mathrm{MHz}$.

For the $8 \mathrm{k}$ mode (used in the rest of Europe), the basic parameters are $T_{U}=896 \mu$ s and $K=6817$. In addition to these basic parameters, others that have to be defined are for modulation, guard interval length and channel coding. For Indoor, Typical Urban and Rural Area measurements, the guard interval lengths of $\tau_{g}=1 / 32 \cdot 896 \mu \mathrm{s}=28 \mu \mathrm{s}$ and $\tau_{g}=1 / 32 \cdot 224 \mu \mathrm{s}=7 \mu \mathrm{s}$ are both suitable because of the maximum channel delay is approximately $5 \mu \mathrm{s}$.

Table III summarizes the transmission parameters used for tests.

\section{EXPERIMENTAL RESULTS}

The system set-up was verified by performing a bench mark test, measuring the receiver's sensitivity in an AWGN channel and comparing it to that published in the DVB-T standard specifications [19].

Subsequently tests were performed as described in Table IV. The dependence of diversity gain against all variables was methodically measured, exploring the effect of varying channel correlation coefficient, diversity delay, Doppler shift on different signal scenarios and in different broadcast modes.

The simulation results reported in the literature suggest an achievable delay diversity gain of approximately 4 to $6 \mathrm{~dB}$ in RA and Indoor channels [11], [20], $3 \mathrm{~dB}$ to $5 \mathrm{~dB}$ in TU [10], [11] and about $1.5 \mathrm{~dB}$ in larger delay spread outdoor channels [7]. The results presented in this section show that the predicted performance can be achieved with actual equipment in simulated signal conditions.

\section{A. Benchmark Test of Experimental Equipment}

This preliminary benchmarking measures the $\mathrm{C} / \mathrm{N}$ for Quasi Error Free (QEF) operation as defined by the DVB-T specifications. The QEF operation is defined as a post-Viterbi Error Rate of $2 \times 10-4$ and this should occur at a $\mathrm{C} / \mathrm{N}$ of 5.9, 12.5 and $16.5 \mathrm{~dB}$ for the predefined 4-QAM UK, UK and French modes respectively. Fig. 5 shows measured BER versus $\mathrm{C} / \mathrm{N}$ for these modes, in this situation it was possible to measure the Post Viterbi BER and $\mathrm{C} / \mathrm{N}$ since an AWGN channel exhibits good stability against time. The sensitivity measured for each mode was within $1 \mathrm{~dB}$ of that published in the DVB specifications confirming the validity of the measurement setup and procedure.

\section{B. Diversity Gain Performance Versus Delay}

This test case aims at evaluating the diversity gain achieved with different delay values in an indoor environment. The cross correlation between the channels was fixed at $\rho=0.25$ and 
TABLE IV

TEST CASES

\begin{tabular}{ccccccc}
\hline \hline TEST CASE & CONFIGURATION & $\delta=[1.5, \ldots, 0.25](\mu s)$ & $\rho=[0.75, \ldots, 0]$ & CHANNEL & $F d=[0, \ldots, 120](H z)$ & PURPOSE \\
\hline A & $\begin{array}{c}\text { UK, } \\
\text { 4AM UK, } \\
\text { French } \\
\text { UK }\end{array}$ & N/A & N/A & AWGN & N/A & $\begin{array}{c}\text { BENCHMARK } \\
\text { WITH DVB-T } \\
\text { SPECIFICATIONS } \\
\text { IMPACT OF } \\
\text { French }\end{array}$ \\
DIFFERENT DELAY \\
VALUES \\
IMPACT OF \\
C
\end{tabular}

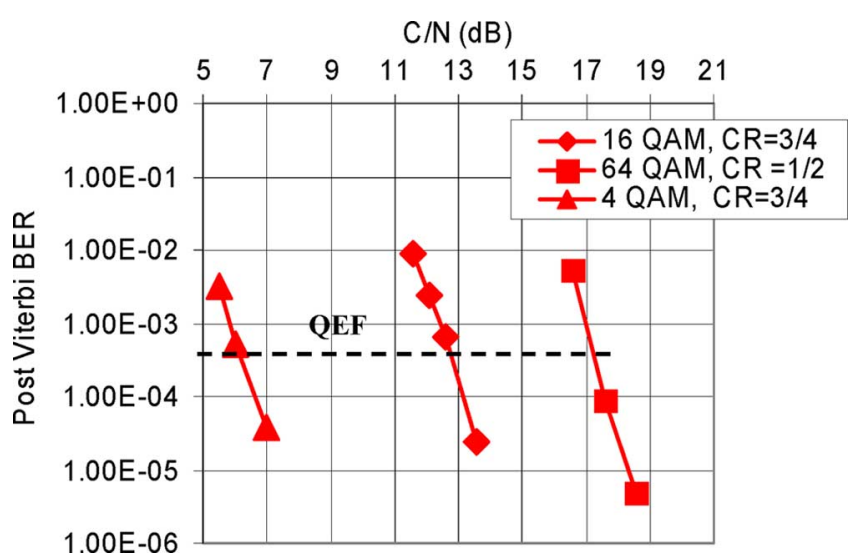

Fig. 5. BER vs. C/N in AWGN channel, UK and French mode.

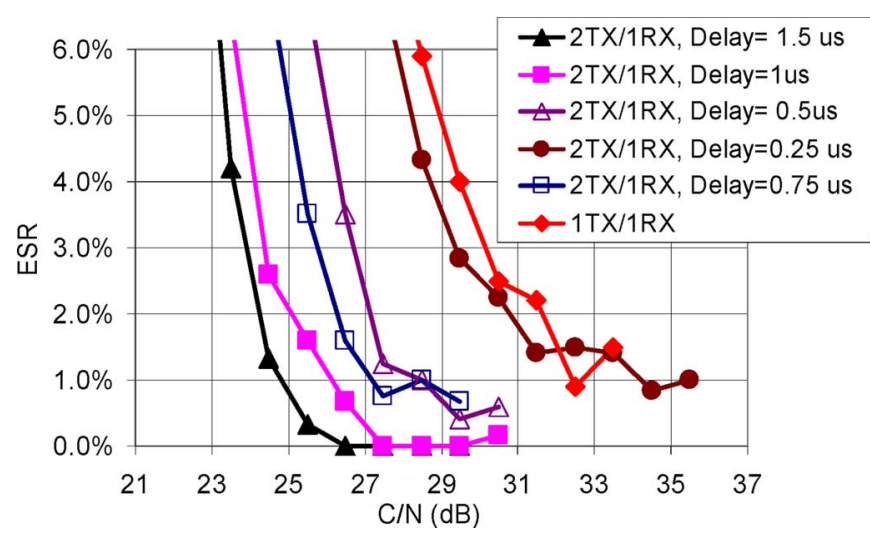

Fig. 6. ESR vs. $\mathrm{C} / \mathrm{N}$ for indoor channel, $\mathrm{UK}$ mode, $\rho=0.25, \mathrm{Fd}=1 \mathrm{~Hz}$.

the Doppler shift was fixed at $1 \mathrm{~Hz}$. Figs. 6 and 7 show ESR

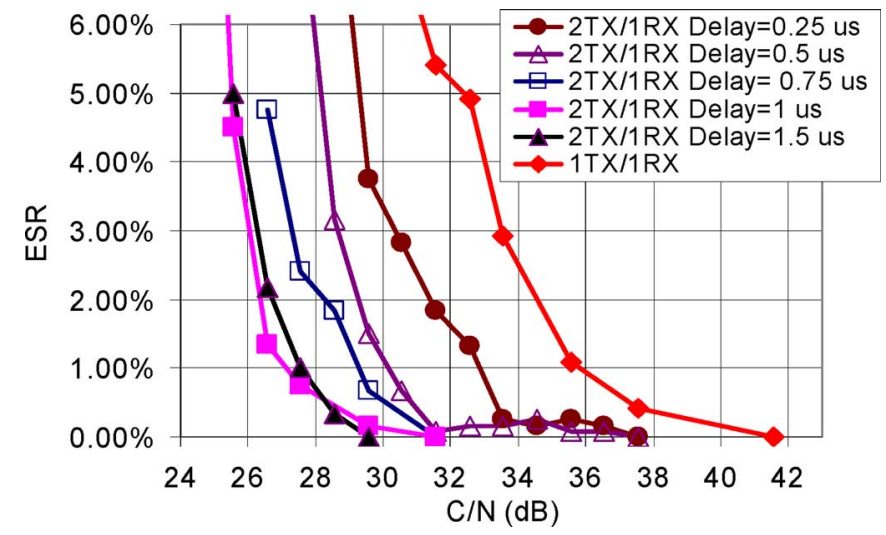

Fig. 7. ESR vs. $\mathrm{C} / \mathrm{N}$ for indoor channel, French mode, $\rho=0.25, \mathrm{Fd}=1 \mathrm{~Hz}$.

versus $\mathrm{C} / \mathrm{N}$ for the indoor channel in the UK and French broadcast modes.

The cross correlation value $\rho=0.25$ corresponds to a distance between the two transmit antennas of about $13 \lambda$. This antenna separation can be reasonably achieved in practical UHF deployments. Single transmit antenna performances are included as a reference. Due to the low correlation coefficient between the channels, subcarriers experiencing a deep fade when transmitted from antenna ' 1 ' are unlikely to experience the same depth of fade when transmitted from antenna ' 2 '. As mentioned in Section II, DD increases channel selectivity and therefore decreases the likelihood of a deep fade occurring due to interference between the signals from the 2 antennas.

Fig. 6 shows results for DD Gain vs. Delay for UK broadcast mode and a simulated indoor channel. This shows that at a delay of $0.25 \mu \mathrm{s}$ and $3 \%$ ESR a gain of $0.7 \mathrm{~dB}$ was achieved compared with the single transmitter case. Furthermore as the delay value increased to $1.5 \mu \mathrm{s}$, the DD gain increased to $5.5 \mathrm{~dB}$.

Similarly for the French mode depicted in Fig. 7, when the delay value is 0.25 us, the DD gain is $3 \mathrm{~dB}$ gain at $3 \%$. As the 


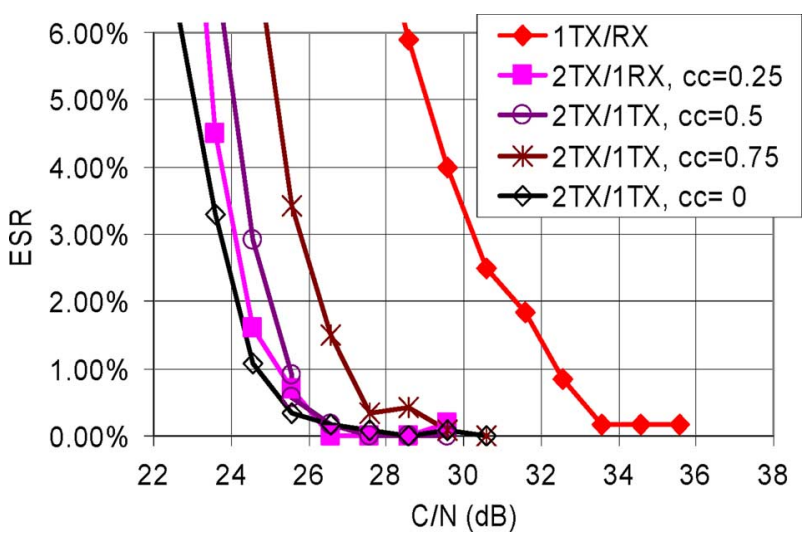

Fig. 8. ESR vs. C/N for indoor channel, UK mode, $\delta=1 \mu \mathrm{s}, \mathrm{Fd}=1 \mathrm{~Hz}$.

delay value increased from to $1.5 \mu \mathrm{s}$, the DD gain increased from to $7.2 \mathrm{~dB}$.

In conclusion, simulations have shown that for indoor scenarios, it can be expected a diversity gain exceeding $5 \mathrm{~dB}$ when the delay between the transmitted signals is above $1 \mu \mathrm{s}$.

\section{Delay Diversity Gain Performance Tests Versus Cross Correlations}

The purpose of this test case is to evaluate the relationship between the cross correlation coefficient between channels and the achievable diversity gain keeping the delay value and Doppler constants. This is extremely important to evaluate since the cross correlation depends on the actual separation of antennas and their configuration in specific channel scenarios. Subsequently field trials will be largely concerned with determining the practical conditions that will produce decorrelated fading.

The results shown in Fig. 8 illustrate the relationship between correlation and diversity gain in the indoor channel with a delay value $\delta=1 \mu \mathrm{s}$ and a Doppler frequency $\mathrm{Fd}=1 \mathrm{~Hz}$ for the UK mode. These results show that when the cross correlation coefficient is less than $50 \%$, a gain of approximately $4 \mathrm{~dB}$ can be achieved and the full gain of over $6.5 \mathrm{~dB}$ could be achieved with the theoretical $0 \%$ correlation. However, it is important to note that in this simulated indoors scenario, the optimal diversity gain can be achieved with a relatively high cross correlation of $50 \%$.

\section{Delay Diversity Gain Tests With a High Doppler Frequency}

Fig. 9 illustrates the effectiveness of diversity in a typical urban (TU) environment at a high Doppler frequency of Fd $=$ $50 \mathrm{~Hz}$. It can be seen that in the SISO case, the ESR value cannot reach $0 \%$ even at high $\mathrm{C} / \mathrm{N}$ ratio. The introduction of a transmit diversity signal with delay of $0.5 \mu \mathrm{s}$ or above provides $5 \mathrm{~dB}$ of diversity gain at an ESR of 3\% and enables $0 \%$ error rate to be achieved.

\section{E. Delay Diversity Gain Tests for Different DVB-T Modes, Maximum Doppler Frequencies and Environments}

Fig. 10 summarizes the diversity gain measured in a number of modes versus delay values. It can be seen that in flat fading environments like indoors, a diversity gain $7.5 \mathrm{~dB}$ for $8 \mathrm{k}$ modes or

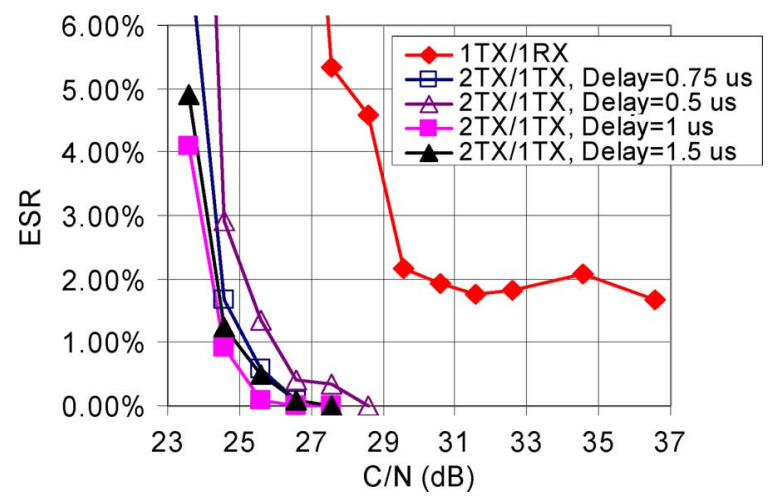

Fig. 9. ESR vs. C/N for TU channel, UK mode, $\rho=0.25, \mathrm{Fd}=1 \mathrm{~Hz}$.

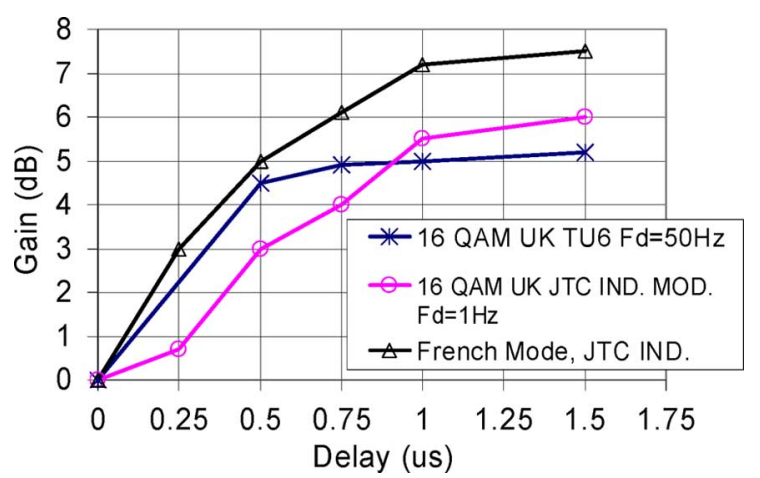

Fig. 10. Delay diversity gain vs. delay code at $\mathrm{ESR}=3 \%, \rho=0.25, \delta=$ $1 \mu \mathrm{s}$.

$6 \mathrm{~dB}$ for $2 \mathrm{k}$ mode can be achieved. The diversity gain is mainly affected by the increase in the delay value. The impact of the modulation or mode of transmission on the achieved diversity gain is not significant as expected from theoretical simulations reported in [7], a delay that is larger than $1.5 \mu$ s results in no further diversity gain improvement.

It was shown in [11] that the achievable gain in the TU channel is $3 \mathrm{~dB}$ less than for the Indoor channel case. This is due mainly to the different channel delay spreads. However, Fig. 10 shows a maximum diversity gain in the TU case comparable with the indoor channel (an offset of about $1 \mathrm{~dB}$ at $\delta=1.5 \mu \mathrm{s}$ ). The reason is mainly due to the Doppler frequency of the TU channel which is $50 \mathrm{~Hz}$ compared with $1 \mathrm{~Hz}$ for the indoor channel and TU6. In [7] a diversity gain of about $2 \mathrm{~dB}$ in TU6 with a low Doppler frequency of $10 \mathrm{~Hz}$ was predicted. This is confirmed by the tests of Fig. 13 in different channels with a Doppler frequency of $1 \mathrm{~Hz}$.

\section{F. Delay Diversity Gain for Different Maximum Doppler Frequencies}

This case studies the impact of Doppler frequency on the achievable diversity gain. The effect of the Doppler frequency on diversity performances of the 4 QAM UK mode is shown in Fig. 11. The 4-QAM is more likely to be adopted for fast mobile receiver applications. This operating mode allows the receiver to be more robust to faster channel variations associated with mobile reception hence providing an acceptable service quality [21]. Fig. 11 shows that the SISO curve diverges from the MISO 


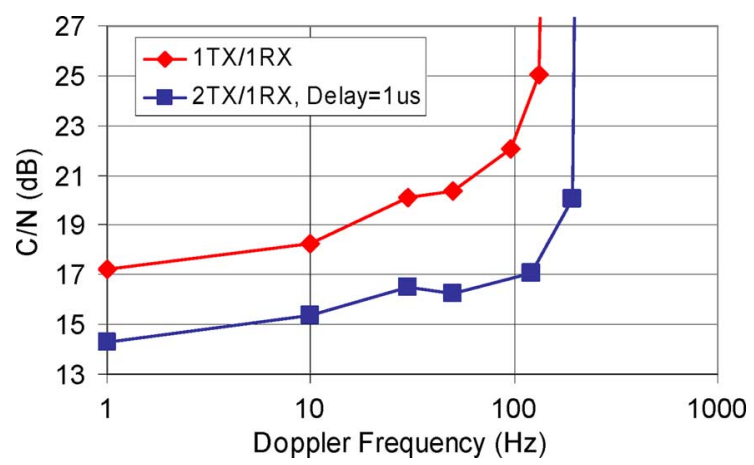

Fig. 11. $\mathrm{C} / \mathrm{N}$ at TOV vs. Doppler frequency, TU, 4-QAM UK mode, ESR = $3 \%, \rho=0.25, \delta=1 \mu \mathrm{s}$.

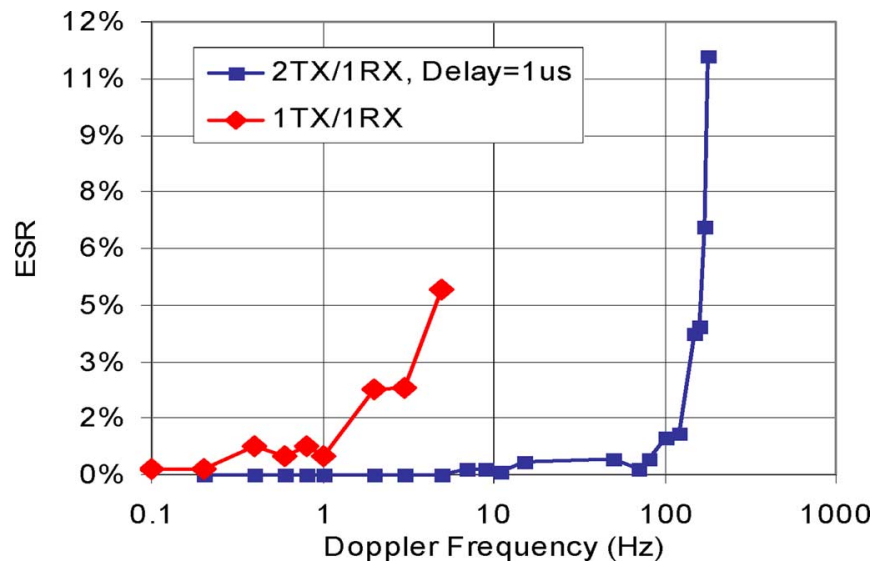

Fig. 12. ESR vs. Doppler frequency at $\mathrm{C} / \mathrm{N}=18.06 \mathrm{~dB}, \mathrm{TU}, 4 \mathrm{QAM} \mathrm{UK}$ mode, $\rho=0.25, \delta=1 \mu \mathrm{s}$.

curve as the Doppler frequency increases. This results in $5 \mathrm{~dB}$ diversity gain at $50 \mathrm{~Hz}$ Doppler frequency in the MISO case.

As the Doppler frequency increases further, the single transmitter curve reaches the failure point faster than the $2 \mathrm{Tx}$ curve. From Fig. 11 it can be observed that for $F d \geq 120 \mathrm{~Hz}$, the receiver cannot track fast channel variations even in the diversity case, resulting in the failure of signal demodulation even with very high $\mathrm{C} / \mathrm{N}$ levels. For low Doppler frequencies $(\sim 1 \mathrm{~Hz})$ as in a quasi-static channel, a diversity gain of approximately $3 \mathrm{~dB}$ was achieved.

Fig. 12 shows the variation of ESR versus Doppler frequency at a fixed $\mathrm{C} / \mathrm{N}$ ratio. With a single Tx the ESR exceeds the 3\% TOV at a low Doppler frequency of around $4 \mathrm{~Hz}$, whereas in the 2 Tx case, the ESR value does not exceed the 3\% threshold until a Doppler of $105 \mathrm{~Hz}$ is reached. For this particular receiver implementation, a Doppler shift of about $105 \mathrm{~Hz}$ introduces sufficient ISI to inhibit the receiver operation.

\section{G. Delay Diversity Tests for Different Environments}

In this test case, the transmit delay diversity gain is evaluated in several environments with different power delay profiles and delay spread values.

Fig. 13 shows the achieved diversity gain in four channels namely BU12, TU6, RA and indoor. All other parameters are kept constant; the cross correlation coefficient is 0.25 , the delay

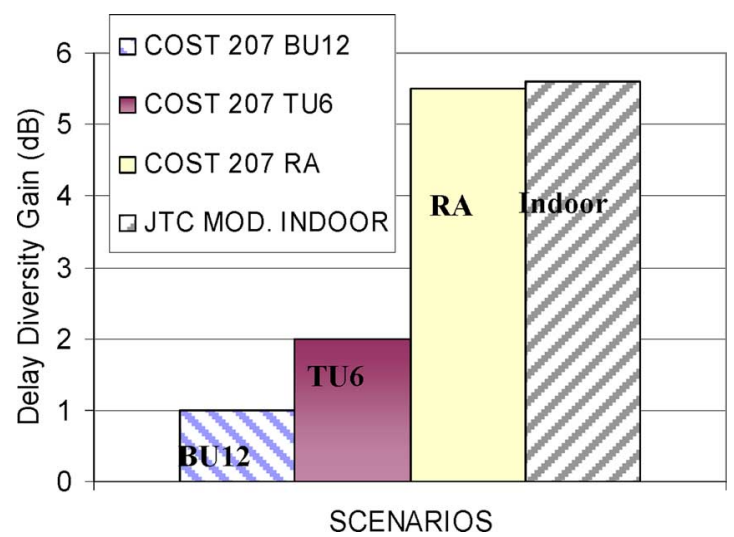

Fig. 13. Diversity gain for different power delay profiles, UK mode, $\rho=0.25$, $\delta=1 \mu \mathrm{s}, \mathrm{Fd}=1 \mathrm{~Hz}$.

value between the channels of the two antennas is $1 \mu \mathrm{s}$ and the Doppler frequency is $1 \mathrm{~Hz}$ in the UK mode.

As expected, channels with large delay spread values lead to smaller diversity gains since there is a low probability of experiencing flat fades. It should be noted that in the case of RA and Indoor channel, the coherence bandwidth is significantly higher than the TU and BU coherence bandwidth. Consequently, a higher number of subcarriers can fall in flat deep fades simultaneously, resulting in a highly demanding channel for the DVB signal. In this situation the greatest diversity gain has been measured showing the potential of transmit diversity in low delay spreads. It is concluded that the diversity gain is inversely proportional to the channel delay spread value.

\section{CONCLUSIONS}

In this paper, the simulated performance of transmit delay diversity has been reported for DVB-T systems. Measurement results confirm that transmit delay diversity (DD) achieves significant diversity gain for de-correlated channels. Diversity gain increases as delay between the transmitted signals increases until there is maximum decorrelation between all received channels. In practice, measurements suggest that a delay value of $1 \mu \mathrm{s}$ should be sufficient to achieve optimal diversity gain. Diversity gains of up to $7.5 \mathrm{~dB}$ should be achievable for indoor scenarios with a low delay spread and for outdoor scenarios with a longer delay spread diversity gains of up to $5 \mathrm{~dB}$ should be achievable. The key factor affecting success of diversity is cross correlation of the channels, measurements have shown that scenarios with a relatively short delay spread can still deliver high diversity gain with relatively high degree of cross correlation at $50 \%$.

For mobile reception, DD significantly improves receiver performance until the impact of Doppler on inter symbol interference becomes unacceptably high for a particular receiver's implementation. It has also been confirmed though practical measurements that the gain predicted form previous theoretical simulations can be realized or exceeded using actual equipment in realistic propagation conditions. The transmit delay diversity can be used to improve significantly the quality of signal of DVB-T/H broadcast networks in inaccessible environments and high speed the receiver situations reducing dependence on diversity receivers. 
Future work will be to perform field tests to verify these conclusions in real world environments and to show how coverage area of a DVB transmitter can be increased by using diversity. It is envisaged that these results should also be applicable for other OFDM broadcast systems.

\section{APPENDIX}

The Dirac delta function is defined as

$$
D(x)= \begin{cases}1 & x=0 \\ 0 & \text { otherwise }\end{cases}
$$

and the sign function is defined as

$$
\operatorname{sgn}(x)= \begin{cases}1 & x>0 \\ 0 & x=0 \\ -1 & x<0\end{cases}
$$

(A.1) and (A.2) are adopted to define

$$
v(x)=\frac{1+\operatorname{sgn}(x)}{2}+\frac{1}{2} D(x)= \begin{cases}1 & x \geq 0 \\ 0 & x<0\end{cases}
$$

which representing a modification of the standard Heaviside step function, where the value in zero is $1 / 2$. If $v(x)$ is shifted by a delay $\delta_{i}, v\left(t-\delta_{i}\right)$ consequently sets zeros for $t<\delta_{i}$.

In (1) the signal code $s_{i}(t)$ is shifted in the time domain at the $i^{t h}$ antenna by delay $\left.\delta_{i}\right)$. The elements of $s_{i}\left(t-\delta_{i}\right)$ having a negative time index (i.e. $t<\delta_{i}$ ), have to be multiplied by zero to comply with the DD codeword definition [22]. This operation can be easily done by multiplying $s_{i}\left(t-\delta_{i}\right)$ by the zeroing function $v\left(t-\delta_{i}\right)$ as shown in (1).

\section{REFERENCES}

[1] “IST Project PLUTO,” [Online]. Available: http://www.ist_pluto.org

[2] T. S. Rappaport, Wireless Communications, Principles and Practice, 2nd ed. New York: Prentice Hall PTR, 2002.

[3] W. C. Lee, Wireless and Cellular Communications. New York: McGraw Hill, 2005.

[4] J.-C. Guey, M. R. Bell, M. P. Fitz, and W.-Y. Kuo, "Signal design for transmitter diversity wireless communication systems over Rayleigh fading channels," in IEEE Vehicular Technology Conf., Atlanta, 1996, pp. $136-140$.

[5] S. Alamouti, "A simple transmitter diversity scheme for wireless communications," IEEE J. Select. Areas Commun., vol. 16, pp. 1451-1458, Oct. 1998.

[6] V. Tarokh, N. Seshadri, and A. Calderbank, "Space-time codes for high data rate wireless communications: Performance criterion and code construction," IEEE Trans. Inform. Theory, vol. 44, pp. 744-765, Mar. 1998.

[7] A. Dammann and S. Kaiser, "Standard conformable antenna diversity techniques for OFDM systems and its application to the DVB-T system," in IEEE GLOBECOM, Nov. 2001, pp. 3100-3105.

[8] M. I. Rahman, K. Witrisal, S. S. Das, F. H. P. Fitzek, O. Olsen, and R. Prasad, "Optimum pre-DFT combining with cyclic delay diversity for OFDM based WLAN systems," in IEEE VTC 2004, May 2004, vol. 4, pp. 1844-1848.

[9] S. B. Weinstein and P. M. Ebert, "Data transmission by frequency division multiplexing using the discrete Fourier transform," IEEE Trans. Commun., vol. 19, no. 15, pp. 628-634, Oct. 1971.
[10] Y. Zhang, J. Cosmas, M. Bard, and Y.-H. Song, "Diversity gain for DVB-H by using transmitter/receiver cyclic delay diversity," IEEE Trans. Broadcasting, vol. 52, no. 4, December 2006.

[11] Y. Zhang, J. Cosmas, K.-K. Loo, M. Bard, and R. D. Bari, "Analysis of cyclic delay diversity on DVB-H systems over spatially correlated channel," IEEE Trans. Broadcasting, vol. 53, no. 1, March 2007.

[12] A. Abdi and M. Kaveh, "A space-time correlation model for multielement antenna systems in mobile fading channels," IEEE J. Selected Areas in Commun., vol. 20, no. 3, pp. 550-560, Apr. 2002.

[13] "Spirent's web page support," [Online]. Available: http://www. spirentcom.com/analysis/technology.cfm?SS $=156 \& \mathrm{WS}=68$

[14] "IST PLUTO PROJECTS—Del 3.2 Backwards Compatibility Testing," [Online]. Available: http://www.ist-pluto.org

[15] "European Telecommunications Standard Institute ETSI, Digital Video Broadcasting (DVB),", Digital Video Broadcasting (DVB), Measurement Guidelines for DVB Systems May 1997, ETR 290.

[16] Joint Technical Committee on Wireless Access, Final Report on RF Channel Characterization JTC(AIR)/93.09.23-238R2, September 1993.

[17] T. Rappaport, Wireless Communications. New York: Prentice Hall, 2002.

[18] COST 207, "Digital Land Mobile Radiocommunications," Office for Official Publications of the European Communities, Abschlussbericht, Luxembourg, 1989.

[19] European Telecommunications Standard Institute ETSI, "Digital Video Broadcasting (DVB),", Framing Structure, Channel Coding and Modulation for Digital Terrestrial Television July 1999, EN 300744 V1.2.1.

[20] "IST PLUTO PROJECTS—Del 3.1a Simulation Lab Facility and Test Report," [Online]. Available: http://www.ist-pluto.org

[21] A. Bertella, M. Rossini, P. Sunna, and L. Vignaroli, Mobile DVB-T Reception: Quality of Streaming Over IP of Audiovisual Services [Online]. Available: http://www.broadcastpapers.com/whitepapers/

[22] C. Oestges and B. Clerckx, MIMO Wireless Communications, 1st ed. Burlington, MA: Elsevier/Academic Press, Jan. 2007.

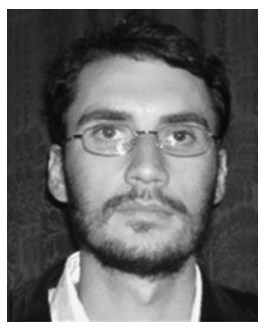

Raffaele Di Bari (M'07) received the B.Sc. and M.Sc. in Telecommunications Engineering in Pisa University, Italy, in 2003 and 2005 respectively. He is currently working toward the Ph.D. degree in the Department of Electrical and Computer Engineering, Brunel University, West London, UK. His current research interests are in the area of Digital Video Broadcasting systems, MIMO-OFDM systems and Radio Channel measurements. Since 2006, he is a Research Assistant with the Networks and Multimedia Communications Group at Brunel University, West London, UK, investigating physical layer optimization of Digital Video Broadcasting (DVB) systems and participating to the IST FP6 PLUTO project. $\mathrm{He}$ is a reviewer for several international conferences. Raffaele Di Bari is a member of the Institute of Electrical and Electronics Engineers (IEEE).

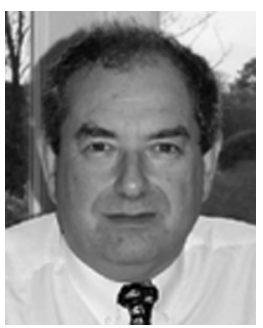

Maurice Bard graduated from Imperial College in 1976 with a BSc (Hon) in Materials Science and worked initially on Travelling Wave Tube design, electronics systems and software. Maurice has succeeded in a number of engineering, sales and marketing roles during a 20 year career at Nortel Networks. Whilst there he founded and managed a business providing GPS Simulators to a world market before moving on to establish a new Fixed Wireless product line which deployed 1 million lines around The World. He left to join PipingHot Networks in 2000; a wireless start-up which is now established as an international provider of Non-Line of Site radio links using similar principles to those proposed here. More recently Maurice has been working as an independent consultant in the wireless, broadcast and GPS industries. 


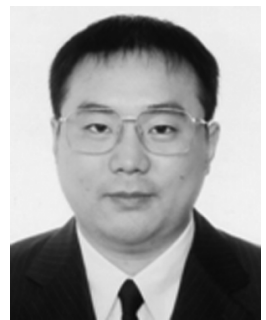

Yue Zhang (M’06) Studied Telecommunications Engineering in Beijing University of Posts and Telecommunications (BUPT) and received B.Eng and M.Eng degrees in 2001 and 2004 respectively. He obtained $\mathrm{PhD}$ degree in electronics engineering at Brunel University in 2008. He had worked for Brunel University as research assistant over two years for IST FP6 PLUTO project, which is to investigate and measure the MIMO effects over DVB-T/H networks. He also designed and implemented the low cost On-Channel repeater in DVB-T/H networks with digital echo cancellation in DSP and FPGA. His research interests are signal processing, wireless communications systems, MIMO-OFDM systems, radio propagation model and multimedia and wireless networks. He currently works in Anritsu Company as signal processing design engineer. He has published over 10 papers in refereed conference proceedings and journals. He also serves as a reviewer for IEEE TRANS. BROADCASTING, WIRELESS COMMUNICATION, CIRCUITS AND SYSTEMS I (CAS I) and guest editor for International Journal of Digital Multimedia Broadcasting.

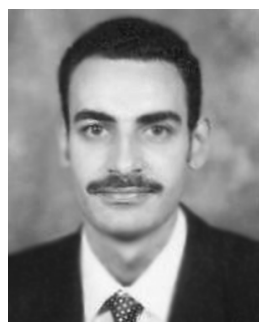

Karim Medhat Nasr received the B.Sc. with Honours and the M.Sc. degrees in Electronics and Communication Engineering from Cairo University, Giza, Egypt in 1995 and 2000 respectively. In 1996, he joined the Egyptian Radio and TV Union (ERTU) as a project engineer working on the design and commissioning of digital broadcasting systems and electronic archiving networks. In September 2001, he was awarded an Overseas Scholarship and joined the Mobile Systems Architecture Group at the University of Manchester, Manchester, UK, from which he received his Ph.D. degree in 2005 on Smart Antenna Systems for Indoor WLAN. Dr. Nasr is presently with BBC Research, British Broadcasting Corporation, UK, investigating future broadcasting systems, media and technologies. He was previously a Research Fellow with the Networks and Multimedia Communications Group at Brunel University, West London, UK, investigating physical layer optimization of Digital Video Broadcasting (DVB) systems. Dr. Nasr was also a Research Associate at the University of Manchester, UK, investigating several projects dealing with future wireless communication systems and applications. During spring 2005, Karim Nasr was a visiting researcher at the antennas and propagation division of Aalborg University, Denmark conducting indoor channel measurements and investigating propagation aspects for future MIMO and UWB systems through a European COST273 sponsored mission. His research interests include propagation modeling and Computational Electromagnetics, Digital Signal Processing (DSP) for future wireless and broadcasting systems, smart antennas and multiple element array processing, multiple user MIMO, OFDM and UWB systems. Dr. Nasr is a reviewer for several transactions and international conferences. Karim M. Nasr is a member of the Institution of Engineering and Technology (IET formerly known as IEE), the Institute of Electrical and Electronics Engineers (IEEE) and European COST Actions 273 and 2100.

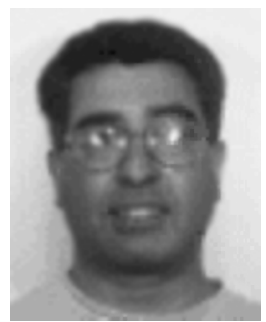

John Cosmas (M'86) obtained a B.Eng honours degree in Electronic Engineering at Liverpool University in 1978 and a PhD in Image Processing and Pattern Recognition at Imperial College, University of London in 1987. He is a Professor of Multimedia Systems and became a Member (M) of IEEE in 1987 and a Member of IEE in 1977. His research interests are concerned with the design, delivery and management of new TV and telecommunications services and networks, multimedia content and databases, and video/image processing. He has contributed towards eight EEC research projects and has published over 80 papers in refereed conference proceedings and journals. He leads the Networks and Multimedia Communications Centre within the School of Engineering and Design at Brunel University.

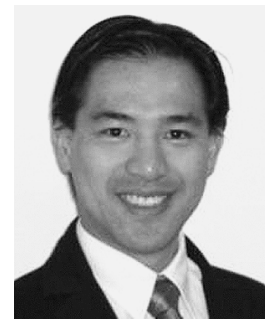

Kok-Keong Loo (M'01) a.k.a. Jonathan Loo received his MSc degree (Distinction) in Electronics at University of Hertfordshire, UK in 1998 and $\mathrm{PhD}$ degree in Electronics and Communication at the same university in 2003. After completing his $\mathrm{PhD}$, he works as a lecturer in multimedia communications at Brunel University, UK. He is also a course director for MSc Digital Signal Processing. Besides that, he currently serves as principle investigator for a joint project between Brunel University and British Broadcasting Corp (BBC) on the Dirac video codec research and development. He also serves as co-investigator for the IST-FP6 PLUTO project. His current research interests include visual media processing and transmission, digital/wireless signal processing, software defined radio, and digital video broadcasting and networks.

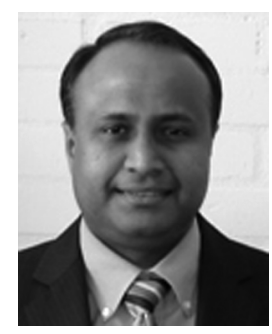

Rajagopal Nilavalan (M'05) received the B.Sc. Eng in Electrical and Electronics Engineering from University of Peradeniya, SriLanka in 1995 and Ph.D in Radio Frequency Systems from University of Bristol, Bristol, UK in 2001. From 1999 to 2005 he was a researcher at the Centre for Communications Research (CCR) at University of Bristol, UK. At Bristol, his research involved theoretical and practical analyses of post reception synthetic focusing concepts for nearfield imaging and research on numerical FDTD techniques. Since 2005, he has been at the Electronics and Computer Engineering department, Brunel university, where he is currently a lecturer. His main research interests include antennas and propagation, microwave circuit designs, numerical electromagnetic modeling and digital video broadcast techniques. Dr. Nilavalan was a member of the European commission, Network of Excellence on Antennas (2002-2005) and a member of the IET.

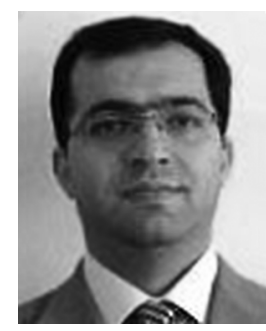

Hamidreza Shirazi (M'04) received his BSc and MSc with honours respectively in Software Engineering from University of Science and Technology, Tehran, Iran in 2000 and Data Communications Systems from School of Engineering and Design, Brunel University, UK in 2004. He is studying for a $\mathrm{PhD}$ in the School of Engineering and Design, Brunel University, UK and contributing to the IST FP6 PLUTO project. His research interests are focused on the quality of service of multimedia services and conditional access systems in converged broadcast, mobile and IP-based networks.

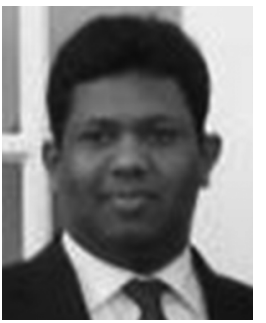

Kannan Krishnapillai received the B.Eng (Hon) First Class Honours in Electrical and Electronics Engineering from Brunel University, West London, UK in 2000 and $\mathrm{PhD}$ in Broadcast Engineering from Brunel University, West London, UK in 2007. From 2000 to 2008 he was a researcher at the Networks and Multimedia Communications Group at Brunel University, UK. At Brunel, his research involved utilization of context specific metadata in converged broadcast and telecommunication systems, design and development of software frameworks for content 